\title{
Sleep Debt Must be Paid Off: Relationship Between Sleep Loss and Mental Health Among Japanese Workers
}

\author{
Akira Mikami*,1,2 ${ }^{,}$Masateru Matsushita ${ }^{3}$, Takuya Watanabe ${ }^{4}$, Emiko Sugiyama ${ }^{5}$, Hiroyoshi \\ Adachi $^{1,2}$, Reiko Matsuura ${ }^{6}$, Yoshiro Sugita ${ }^{1,2}$ and Masatoshi Takeda ${ }^{2}$ \\ ${ }^{1}$ Osaka University Health Care Center, Toyonaka, Osaka \\ ${ }^{2}$ Psychiatry, Department of Integrated Medicine, Division of Internal Medicine, Osaka University Graduate School of \\ Medicine, Suita, Osaka \\ ${ }^{3}$ Department of Psychiatry and Neuropathobiology, Kumamoto University Graduate School of Medical Science, Kuma- \\ moto, Japan \\ ${ }^{4}$ Kosaka Hospital, Higashi Osaka, Osaka, Japan \\ ${ }^{5}$ Osaka Prefectural Medical Examiner's Office, Osaka, Japan \\ ${ }^{6}$ Osaka Prefectural Mental Health Center, Osaka, Japan
}

\begin{abstract}
Background: Sleep loss and sleep debt are common in Japanese workers. Although cumulative sleep debt may be considered a cause of mental health problems, that relationship has not been empirically confirmed. Sleep loss is defined as either self-perceived insufficient sleep and/or insomnia. We calculated sleep debt by subtracting the average number of hours of weekday sleep from hours slept on weekends.

Methods: This cross-sectional study was conducted from April 2005 through March 2007 at the Osaka Prefectural Mental Health Center. Participants were 873 workers. First, we investigated characteristics of individuals with awareness of sleep loss. Second, we examined whether reasons for sleep loss (insufficient sleep or insomnia) were associated with sleep habits and psychometric variables. Finally, we identified characteristics of subjects with insufficient sleep and examined the association between self-perception of insufficient sleep and mental health.

Results: Of the subjects, $64.0 \%$ perceived their own sleep loss. Workers with sleep loss related to overwork had significantly larger sleep debt. Subjects with predictable symptoms of insufficient sleep (short sleep on weekdays, difficulty in awakening or sleep maintenance, large sleep debt, and daytime sleepiness) tended to have higher scores on the General Health Questionnaire-28.

Conclusion: Short sleep duration ( $<6$ hours/weekday), insomnia, and insufficient sleep are different although they can overlap and must be considered separately. Sleep debt might be an essential and useful indicator of insufficient sleep especially caused by overwork. Assessing sleep health, including sleep debt, is important for promoting mental health. We postulate that sleep debt must be paid off.
\end{abstract}

Keywords: sleep debt, insufficient sleep, insomnia, sleep loss, mental health, workers.

\section{INTRODUCTION}

Workers in developed countries confront acute and/or cumulative shortage of sleep time. In Japan, it was reported that among the general adult population $29 \%$ slept less than 6 hours a night, 23\% had self-perceived insufficient sleep, $6 \%$ took sleep-enhancing medications, $21 \%$ had symptoms of insomnia, and $15 \%$ had symptoms of excessive daytime sleepiness [1]. Kaneita et al. reported that $13.7 \%$ of their study population slept for less than 6 hours and that $33.3 \%$

\footnotetext{
*Address correspondence to this author at the Psychiatry, Department of Integrated Medicine, Division of Internal Medicine, Osaka University Graduate School of Medicine, Yamada-oka 2-2, D3, Suita, Osaka 565-0871, Japan; Tel: +81-6-6879-3051; Fax: +81-6-6879-3059;

E-mail: mikami@psy.med.osaka-u.ac.jp
}

had insufficient sleep, which put them at high risk of excessive daytime sleepiness [2].

Compared with those in Western nations, Japanese workers are forced to work long hours, possibly due to their cultural background, even though work hours tend to be shorter than in the past [3]. Overwork has adverse health effects and occasionally may contribute to suicide [4]. To prevent death from overwork, 'Karoshi', the Ministry of Health, Labor and Welfare of Japan recommended that a worker not work more than 45 hours overtime per month [5]. Furthermore, the Industrial Safety and Health Law mandates that employers must make arrangements for employees who have worked overtime exceeding 100 hours per month be examined for psychological and physical health by a physician [6]. 
These National commitments were created in response to a societal need. However, despite these efforts, workers who are forced to work overtime generally reduce sleep time on weekdays and are prone to sleep longer on weekends [7]. This disparity between weekday and weekend sleep duration has been regarded as sleep debt. In the only examination of sleep debt of white-collar workers in Japan, Kageyama et al. investigated whether sleep debt correlates with workload and reported that the mean \pm SD of sleep debt on weekdays, which they defined as the difference between self-perceived required sleep length and actual sleep length on weekdays, was $45.1 \pm 65.3 \mathrm{~min}$ and that the workload score and extensive overtime were independently associated with sleep debt accumulated during weekdays in workers from age 20 to 29 years [8].

Although the negative impact of sleep debt is widely recognized, additional research is needed to further understand the adverse psychological consequences of sleep loss, either due to insufficient sleep or insomnia, and sleep debt. However, problems related to sleep needs have not been clearly defined, and there is a wide range of individual differences in sleep needs. Léger et al. reported that persons designated as having short sleep duration, defined as sleeping less than 6 hours per weekday, do not constitute a homogeneous group and include both insomniacs and individuals with or without sleep debt [9].

Our study aims to reveal the frequency of and reasons for sleep loss and to examine the association between sleep debt and its related factors, including effects on both mental and physical health, in a sample of workers. In our analysis, we defined 'short sleep duration' as less than 6 hours of sleep per weekday, 'insomnia' as the subjective report of sleeping difficulties (poor quality of sleep), 'insufficient sleep' as less than the required sleep time (poor quantity of sleep) and 'sleep debt' as the cumulative effect of insufficient sleep assessed by subtracting the average number of hours slept on weekdays from hours slept on weekend days. This definition, incidentally, differs from that of Kageyama et al. Moreover, 'sleep loss' refers in general to poor quantity and/or quality of sleep (insufficient sleep and/or insomnia).

Because insufficient sleep that causes sleep debt and insomnia can overlap although they are different entities, we considered that pure insufficient sleep without insomnia and pure insomnia without insufficient sleep must be compared and examined separately. First, we investigated important characteristics of individuals who were aware of sleep loss compared with those who perceived that they had sufficient sleep. Second, to explore sleep loss in detail, we examined whether reasons for sleep loss (insomnia or insufficient sleep) were associated with sleep habits and psychometric variables. Finally, we identified the characteristics of those with subjective feelings of insufficient sleep after excluding insomnia from sleep loss to determine the variables that could be appropriate discriminators for insufficient sleep. We, furthermore, aimed to examine the association between self-perception of insufficient sleep and mental health.

\section{MATERIALS AND METHODOLOGY}

\section{Participants}

This study is part of research that has been organized at the Division of Stress Management of the Osaka Prefectural Mental Health Center, which is a governmental office, not a medical facility. Participants were enrolled from April 2005 through March 2007 at the Osaka Prefectural Mental Health Center and were selected among the 873 individuals who participated in a mental health program and underwent an examination of the state of their mental health. This was not a treatment program, but rather a program to foster mental health. Most of the 873 examinees were employees of companies or public offices that contracted with the Osaka Prefectural Mental Health Center as part of mental health promotion measures for their employees. As part of this program, they were interviewed by a psychologist regarding life events and awareness of stress and a psychiatrist for a possible diagnosis of mental illness. Blood tests and an electroencephalogram were part of the physical examination. They also completed a questionnaire related to mental and physical health.

Of the 873 initial participants, 87 who were under treatment for a mental disorder or were thought to need such treatment through an interview by a psychiatrist were excluded from analysis. Further exclusion criteria were 1) unemployment $(n=19)$ and 2) engaging in shift work $(n=42)$. Finally, 725 workers, age 20-65 years [mean age 43.2 years $(\mathrm{SD}=10.0)$; 344 men (mean 44.9 years) and 381 women (mean 41.6 years)], were included in the statistical analysis (Fig. 1).

We obtained written informed consent from all study participants whose data were used. The study protocol was approved by The Osaka University Health Care Center Ethical Committee.

\section{Measurements}

The questionnaire was comprised of the following 6 sections: 1) demographic characteristics, 2) questions about awareness of sleep loss and reasons for it, 3) Pittsburgh Sleep Quality Index (PSQI), 4) Epworth Sleepiness Scale (ESS), 5) General Health Questionnaire-28 (GHQ), and 6) Sleep Health Risk Index (SHRI).

Regarding characteristics, information was obtained on gender, age, height, weight, and whether work involved shift work. Perception of sleep loss was determined by the answer to the following question: 'Do you think that presently you are getting enough sleep?' There were 4 possible responses: 1) It is enough; 2) I feel the lack of sleep and want more sleep; 3) Since I am getting too much sleep, I want to shorten my sleep time, and 4) None apply to me. Since the choice of 2) indicated a perception of sleep loss, we asked such responders to select one of the following reasons: 1) Can't sleep due to psychological distress (Psychological Distress Group); 2) Frequent arousal during sleep due to physical problems, such as going to the lavatory frequently (Physical Problem Group); 3) Wake up frequently because of raising children or providing nursing care (Nursing Care Group); 4) 


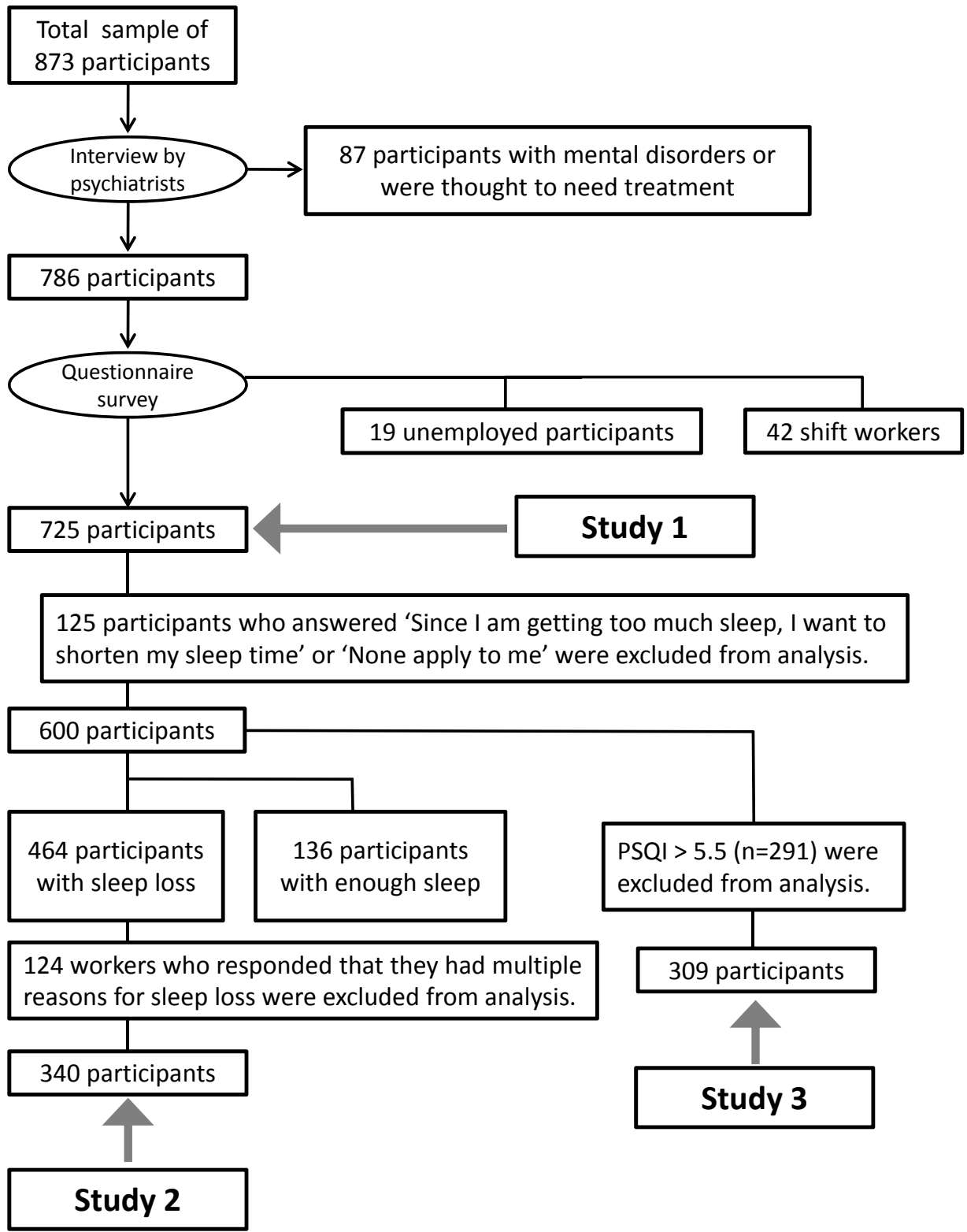

Fig. (1). Schematic of flow of participants from the initiation of the study

There were 725, 340, and 309 participants in Study 1, Study 2 and Study 3, respectively.

Stay up late to engage in a hobby (Hobby Group); 5) Cannot get sufficient sleep time because of long workday or commute to office (Overwork-related Group); and 6) other reasons.

The PSQI was developed by Buysse et al. [10] in 1987 to assess subjective quality of sleep and was translated into Japanese by Doi et al. [11]. The Japanese version of PSQI has good reliability and validity. This scale consists of 18 items, and the global score ranges from 0 to 21, with higher scores representing poor quality of sleep. It was shown that the optimal cutoff score in the Japanese version of PSQI, which maximizes both sensitivity and specificity, is 5.5 [11]. The ESS indicates excessive daytime sleepiness and has a maximum score of 24 . This scale is widely used for assessing sleepiness in patients with sleep disorders [12]. The GHQ-28 is a 28-item measure that assesses concerns about psychiatric symptoms, with responses on a 4-point Likert scale with scores that can range from 0 to 28. Higher scores reflect poorer psychological well-being of the respondent [13]. The SHRI is a 14-item measurement of the degree of sleep health and is comprised of five subscales that were determined by factor analysis: 1) sleep maintenance problems, 2) parasomnia-like problems, 3) sleep apnea, 4) difficulty in waking up, and 5) difficulty in initiating sleep [14].

\section{Procedures}

\section{Study 1: Characteristics of Sleep Loss in Comparison with Sufficient Sleep}

As shown in Fig. (1), after excluding participants in the mental health program from our analysis for various reasons, 725 regular daytime workers without mental disorders were 
eligible for Study 1. Perception of sleep loss was determined as described above. After excluding participants who answered 'Since I am getting too much sleep, I want to shorten my sleep time' or 'None apply to me', we selected as subjects with sleep loss those who answered 'I feel the lack of sleep and want more sleep' and those with sufficient sleep as those who answered 'It is enough'. We compared sleep time on weekdays and weekend days and scores on the PSQI, ESS, and GHQ between subjects with awareness of sleep loss and those with sufficient sleep.

\section{Study 2: Reasons for Sleep Loss (Insufficient Sleep or In- somnia)}

Nursing Care, Hobby and Overwork-related Groups were regarded as having insufficient sleep and the Psychological Distress and Physical Problem Groups were regarded as having insomnia. Insufficient sleep (causing sleep debt) and insomnia can overlap although they are different entities. To compare pure insufficient sleep without insomnia and pure insomnia without insufficient sleep, subjects who reported multiple reasons for sleep loss were excluded from the analysis in Study 2 (Fig. 1).

\section{Study 3: features of insufficient sleep}

The purpose of Study 3 was to reveal the features of those who subjectively perceived insufficient sleep. To distinguish sleep loss by insufficient sleep as being different from insomnia, we eliminated workers from this analysis with the global score on the PSQI at or above the cut-off value (5.5) corresponding to poor quality of sleep (Fig. 1). To identify the features of those with awareness of sleep loss due to insufficient sleep (poor quantity of sleep) after excluding subjects with insomnia (poor quality of sleep), we conducted canonical discriminant analysis. We aimed to identify whether groups with/without insufficient sleep could be separated statistically and which variables were the appropriate discriminators. Lastly, we examined the association between self-perception of insufficient sleep and mental health.

\section{Statistical analysis}

We compared continuous scores using an unpaired $t$ test and one-way analysis of variance (ANOVA). With regard to methods for multiple comparison, a post-hoc Sidak test was performed. To identify whether the groups with/without insufficient sleep could be separated statistically and which variables were appropriate discriminators, we conducted a stepwise discriminant analysis with the Wilks' Lambda method. The criterion for inclusion/exclusion of the $P$ value was 0.05. The SPSS (SPSS, Tokyo, Japan) for Windows 15.0 was used for the entire data analysis. All tests were twotailed at a significance level of 0.05 .

\section{RESULTS}

\section{Study 1: Characteristics of Sleep Loss in Comparison with Sufficient Sleep}

Of 725 subjects who responded to the question regarding sleep loss, 464 (64.0\%) perceived their own sleep loss and 136 (18.8\%) responded 'it is enough' indicating no sleep loss. In comparing the 136 participants who indicated that they got enough sleep with the 464 participants with subjective sleep loss, the latter group had shorter sleep duration on weekdays and weekend days, larger sleep debt, higher PSQI, and increased ESS and GHQ scores (Table 1). The mean sleep debt of individuals with sleep loss (insufficient sleep and/or insomnia) was 86.6 minutes. The prevalence of short sleep duration defined as sleeping less than 6 hours per weekday among subjects with sleep loss was 32.8\%.

\section{Study 2: Reasons for Sleep Loss (Insufficient Sleep or In- somnia)}

To look closely at sleep loss, we addressed the reasons for sleep loss after excluding subjects who reported multiple reasons for sleep loss $(n=124)$. The Nursing Care Group consisted of only 5 participants and 85 subjects responded "other reasons"; therefore, subjects in these groups were excluded from analysis. Finally, 4 groups were analyzed. The Psychological Distress Group ( $\mathrm{n}=52)$ and Physical Problem Group ( $n=30)$ were considered to be groups with poor quality of sleep (insomnia). The Hobby Group $(\mathrm{n}=64)$ and Overwork-related Group ( $\mathrm{n}=104)$ were considered to be groups with poor quantity of sleep (insufficient sleep). Results of the analysis of the 4 groups are shown in Table 2.

There were no significant differences in weekend sleep time and ESS among the 4 groups. Sleep duration on weekdays was longer in the Physical Problem Group than in the

Table 1. Comparisons of Sleep Habits and Psychometric Variables between Subjects with Sleep Loss and those with Sufficient Sleep

\begin{tabular}{|c|c|c|c|c|c|c|}
\hline & \multicolumn{2}{|c|}{ Sleep Loss } & \multicolumn{2}{|c|}{ Sufficient Sleep } & $t$ & $\boldsymbol{P}$ \\
\hline weekend days & 446.4 & (80.61) & 476.0 & (70.87) & 4.15 & $<0.001$ \\
\hline Sleep debt & 86.6 & (75.34) & 55.8 & (61.90) & -4.84 & $<0.001$ \\
\hline ESS & 7.0 & (3.39) & 5.6 & (2.76) & -4.91 & $<0.001$ \\
\hline GHQ & 9.8 & (6.01) & 6.0 & (5.57) & -6.84 & $<0.001$ \\
\hline
\end{tabular}

PSQI: Pittsburgh Sleep Quality Index, ESS: Epworth Sleepiness Scale, GHQ: General Health Questionnaire-28 
Psychological Distress Group and Overwork-related Group. Sleep debt was large in the groups with poor quantity of sleep (insufficient sleep) and was significantly larger in the Overwork-related Group than in the groups with poor quality of sleep (insomnia). Although scores of the PSQI and GHQ were significantly higher in the Psychological Distress Group compared with the other groups, these scores were significantly lower in the Hobby Group than in the poor quality of sleep groups. The prevalence of short sleep duration defined as sleeping less than 6 hours per weekday in the Overwork-related Group was $40.4 \%$.

\section{Study 3: Features of Insufficient Sleep}

To identify the features of participants with awareness of insufficient sleep (poor quantity of sleep) from sleep loss, after excluding those with insomnia according to the PSQI score, we conducted a canonical discriminant analysis (Table 3). The results showed that compared to workers with enough sleep, those with awareness of insufficient sleep were characterized by a short sleep time on weekdays, difficulty in waking up, large sleep debt, difficulty in sleep maintenance, and a high level of daytime sleepiness. Difficulty in waking up (0.44) and sleep debt (0.32) had the largest effects after short sleep time on weekdays $(-0.77)$.

Then, in Table 4, we show a $2 \times 2$ contingency table of correct and incorrect predictions of group membership results from discriminant analysis. As can be seen in Table 4, $72.8 \%$ could be correctly classified, and the total sample of 2 groups was divided into 4 groups. Group $A(n=89)$ did not have subjective awareness of insufficient sleep nor the characteristic symptoms of insufficient sleep. Group $B(\mathrm{n}=60)$ had awareness of insufficient sleep but not symptoms. Group $C(\mathrm{n}=24)$ did not have awareness of insufficient sleep but did have symptoms and Group $D(\mathrm{n}=136)$ had both awareness of insufficient sleep and the predictable symptoms of insufficient sleep. Groups $B$ and $D$ (having subjective awareness of insufficient sleep) had significantly higher total scores on the GHQ than Group A. To investigate the mental health of workers who did not perceive insufficient sleep subjectively but were thought to have insufficient sleep objectively, we compared the GHQ scores of Group $C$ with Group A. Although the mean GHQ score was higher in Group $C$ than in Group $A$, a post-hoc test revealed no significant difference (Fig. 2).

\section{DISCUSSION}

Research that focuses not on 'insomnia' but on 'insufficient sleep' is still sparse. There are several reasons for this. Among them are that 1 ) because there are individual differences in needed sleep time, hours of sleep may not reflect insufficient sleep completely; 2) because both insufficient sleep and insomnia induce short sleep and/or sleep loss secondarily, physicians may confuse insomnia with insufficient sleep in an individual; and 3) because chronic insufficient sleep might cause insomnia, some patients might have both insufficient sleep and insomnia. Moreover, not all individuals who have sleep loss perceive their own sleep loss.

The purposes of this study were to clarify the prevalence of awareness of sleep loss, whether due to insufficient sleep and/or insomnia, and to examine closely the association between reasons for sleep loss and its related factors in Japanese workers who appeared to be healthy. We, furthermore,

Table 2. Comparisons in Sleep Habits and Psychometric Variables According to Reasons for Sleep Loss.

\begin{tabular}{|c|c|c|c|c|c|c|c|c|c|c|c|}
\hline & \multicolumn{2}{|c|}{ Psychological distress ${ }^{a}$} & \multicolumn{2}{|c|}{ Physical problem $b$} & \multicolumn{2}{|c|}{ Hobby ${ }^{c}$} & \multicolumn{2}{|c|}{ Overwork-related $d$} & $\boldsymbol{F}$ & $P$ & Post-hoc test * \\
\hline weekend days & 423.5 & $(98.10)$ & 445.0 & $(63.56)$ & 454.7 & $(69.74)$ & 455.2 & $(71.60)$ & 2.26 & 0.082 & N.A \\
\hline Sleep debt & 69.2 & $(84.76)$ & 53.5 & $(53.10)$ & 91.3 & $(75.48)$ & 109.6 & $(66.45)$ & 6.67 & $<0.001$ & $a<d, b<d$ \\
\hline ESS & 6.6 & $(3.42)$ & 7.6 & (3.57) & 6.2 & $(3.00)$ & 7.4 & (3.33) & 2.15 & 0.094 & N.A \\
\hline GHQ & 14.3 & (5.69) & 9.3 & $(5.76)$ & 6.2 & $(4.84)$ & 8.3 & $(4.97)$ & 24.68 & $<0.001$ & $b, c, d<a, c<b$ \\
\hline
\end{tabular}

"Sidak test, $P<0.05 ;$ N.A, not applied.

PSQI: Pittsburgh Sleep Quality Index, ESS: Epworth Sleepiness Scale, GHQ: General Health Questionnaire-28

Table 3. Standardized Canonical Discriminant Coefficients of Variables that Predict Subjective Insufficient Sleep

\begin{tabular}{|l|c|c|}
\hline \multicolumn{1}{|c|}{ Predictor of Insufficient Sleep } & Standardized Canonical Discriminant Coefficient & Wilks' $\lambda$ \\
\hline \hline Sleep time on weekdays & -0.77 & 0.84 \\
\hline Difficulty in waking up & 0.44 & 0.75 \\
\hline Sleep debt & 0.32 & 0.73 \\
\hline Difficulty in sleep maintenace & 0.31 & 0.73 \\
\hline Epworth Sleepiness Scale score & 0.22 & 0.72 \\
\hline
\end{tabular}




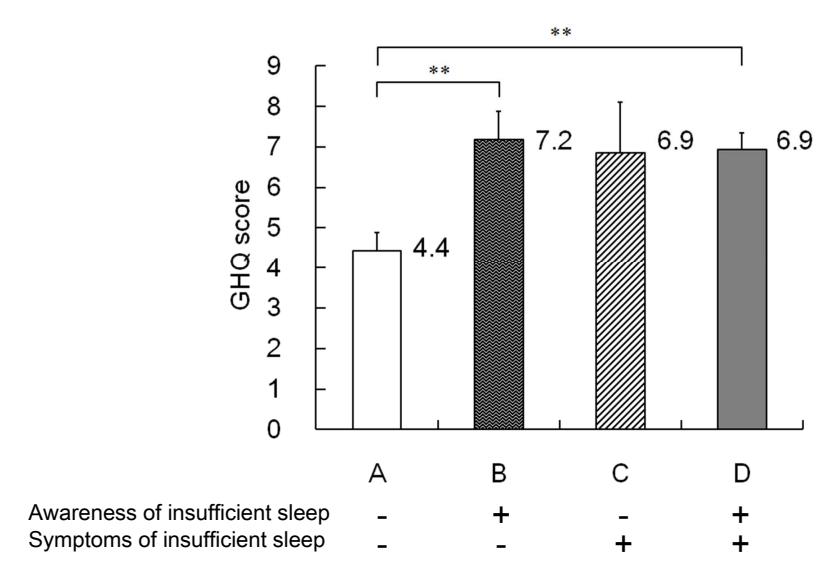

Fig. (2). Sidak multiple comparisons of General Health Questionnaire (GHQ) scores of four groups of participants

Data shown are means \pm SEM. **: $P<0.01$.

A: no sleep loss, B: similar to hypochondria, C: like behaviorally induced insufficient sleep syndrome, D: subjective and objective insufficient sleep

identified features of those with awareness of insufficient sleep after excluding those with insomnia and investigated the mental health of workers who did not perceive having insufficient sleep but were thought to have symptoms indicating insufficient sleep.

Results of Study 1 showed that $64.0 \%$ of 725 regular daytime workers without mental disorders perceived their own sleep loss, that is, they had either insufficient sleep, insomnia or both. This rate was very high compared with results of other studies [1,2], possibly owing to specific characteristics of the participants in this study as mentioned below. However, results showing that individuals with sleep loss had shorter sleep duration on weekdays and weekend days, larger sleep debt, and higher PSQI, ESS and GHQ scores than those with sufficient sleep corresponded with results of previous studies [1,14-16].

In Study 2, we compared sleep habits and psychometric variables according to reasons for sleep loss. The number of participants with insufficient sleep (Hobby or Overworkrelated Group) was twice that of those with insomnia (Psychological Distress or Physical Problem Group). Sleep debt was larger in the insufficient sleep groups compared with the insomnia groups and was significantly larger in the Overwork-related Group (mean $109.6 \mathrm{~min}$ ). PSQI and GHQ scores were significantly higher in the Psychological Distress Group than in the other three groups. Therefore, we concluded that insufficient sleep and insomnia are different and must be distinguished both for purposes of research and treatment. Sleep debt might be a good indicator of the degree of insufficient sleep, especially that caused by overwork.

Study 3 identified the features of insufficient sleep after differentiating insufficient sleep from insomnia by the PSQI score. Our results indicated that short sleep duration on weekdays is the most important factor in insufficient sleep. However, those with short sleep duration do not comprise a homogeneous group and include both insomniacs and subjects with or without sleep debt [9]. Unfailingly, sleep debt is considered to be a very important factor in assessing insufficient sleep.

The discriminant analysis in Study 3 correctly reclassified $72.8 \%$ of the workers in the 4 groups (Table 4), but, likewise, these factors misclassified $27.2 \%$ of the workers in the 4 groups. These reclassified groups were labeled as Group A (no sleep loss), Group B (similar to hypochondria), Group $C$ (like behaviorally induced insufficient sleep syndrome), and Group $D$ (subjective and objective insufficient sleep). Group $C$ did not have subjective awareness of insufficient sleep but had the predictable symptoms of insufficient sleep. Although not significantly, the mean GHQ score was higher in Group $C$ than in Group A. Lack of subjective awareness of insufficient sleep was notable and attention should be paid to this phenomenon when assessing sleep health, including sleep debt. Such a determination could be very important for promotion of mental health.

The present study has several limitations. First, the participants in this study were subjected to a thorough medical checkup for stress at the Osaka Prefectural Mental Health Center. Although we excluded participants who were under treatment or were thought to need treatment for mental disorders, the rate of sleep loss (64.0\%) in Study 1 seems very high. However, results of Study 2 revealed that the number of participants with insufficient sleep was twice as great as that with insomnia as the cause of sleep loss. Second, we analyzed data on the participants after distinguishing insufficient sleep (poor quantity of sleep) from insomnia (poor quality of sleep) in this study. Therefore, we could not analyze characteristics of participants who reported multiple reasons for sleep loss, which suggested that they had both insufficient sleep and insomnia and that their sleep loss was from overlapping causes. Finally, in the present study, we assessed 'sleep debt' by subtracting the average number of hours slept on weekdays from hours slept on weekend days. Regestein et al. defined sleep debt as total sleep time respondents estimated that they needed to be at their best minus the self-reported interval between usual weekday bed-

Table 4. Prediction of Group Membership Based on Discriminant Analysis

\begin{tabular}{|c|c|c|c|c|c|}
\hline \multirow{2}{*}{ Actual Group } & \multirow{2}{*}{ No. Cases } & \multicolumn{3}{|c|}{ Predicted Group Menbership } \\
\cline { 3 - 6 } & & \multicolumn{2}{|c|}{ Group 1 } & \multicolumn{2}{|c|}{ Group 2 } \\
\hline \hline Group 1: Insufficient sleep - & 113 & 89 & $(78.8 \%)^{A}$ & $(21.2 \%)^{C}$ \\
\hline Group 2: Insufficient sleep + & 196 & 60 & $(30.6 \%)^{B}$ & 136 & $(69.4 \%)^{D}$ \\
\hline \multicolumn{2}{|c|}{ Percentage of correct classification } & & \multicolumn{2}{|c|}{$72.8 \%$} \\
\hline
\end{tabular}




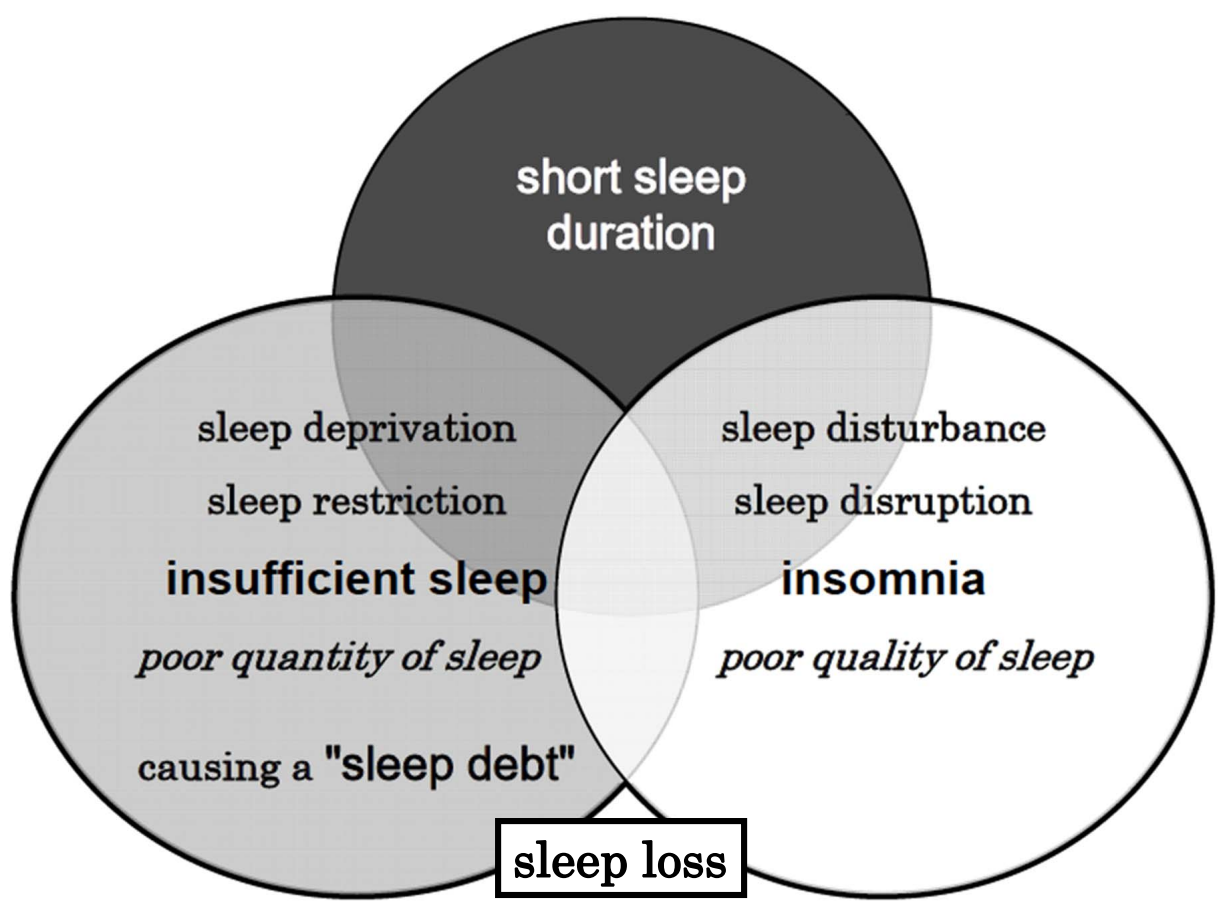

Fig. (3). Short sleep duration, insomnia and insufficient sleep

Sleep loss represents poor quantity and/or quality of sleep with or without short sleep.

time and rising time [17]. However, Prather et al. measured sleep debt by the same method as we did and reported that this measure of sleep debt is based on the assumption that individuals attempt to recoup lost sleep during the weekdays on weekend days [18]. We felt that this method could not be used for shift workers, who were excluded from the analysis.

We cite information that was extracted regarding the 'suicide prevention campaign' in Japan from the Weblog of 'JapanVisitor.com' as shown below [19]. The writer of this blog is unknown.

"According to the National Police Agency, over 30,000 people in Japan have killed themselves each year over the past 14 years, from 1998 to 2011. The Cabinet Office of the Government of Japan considered that an inability to sleep that lasts over two weeks is an important clue that someone may be depressed and started a suicide prevention campaign focusing on the issue of insomnia, called "Nemuretemasuka?" or "Are you sleeping OK?" The message is "If you haven't been sleeping properly for two weeks or more, you may be depressed and liable to commit suicide.” Consultation with a doctor is recommended for people in this condition."

Although consultation with a doctor is recommended for people in this condition, hypnotics and/or antidepressants may be the only treatment given. We must pay more attention to sleep hygiene, including examination of sleep debt, for maintaining good sleep and good mental health. In fact, Kohyama hypothesized that more sleep would induce production of more serotonin and result in fewer suicides in Japan [20].

Acute experimental sleep restriction for the duration of a work week was reported to impair performance and to have the potential for reducing psychological adjustment or fur- ther exacerbating psychiatric illness, although individual differences were large [21]. Increasing evidence suggests an association between short sleep duration and various comorbidities (weight gain [22], diabetes mellitus [23], hypertension [24], cardiovascular disease [25, 26]) and higher mortality [27]. The dissemination of knowledge of adequate sleep hygiene, especially the necessity of sufficient sleep, is considered essential to an employee's welfare and to prevent the development of health problems.

In this study, we investigated sleep habits and psychological variables according to whether sleep loss was due to insufficient sleep or insomnia. It should be considered that short sleep duration, insomnia, and insufficient sleep are different although these conditions can overlap, but that insufficient sleep may be more crucial and essential to an individual's well being than short sleep duration and insomnia. The number of subjects with insufficient sleep was higher than that with insomnia, and the sleep debt in the Overworkrelated Group was significantly larger than in the insomnia groups. Mental health was significantly worse in the subjects with sleep loss, especially in the Psychological Distress Group (an insomnia group) as might be expected. However, it is noteworthy that not only subjects who were aware of insufficient sleep but also subjects who had symptoms indicating insufficient sleep who did not recognize that they were getting sufficient sleep tended to be in poor mental health.

Our results showed $67.2 \%$ of subjects with sleep loss (insufficient sleep and/or insomnia) in Study 1 and 59.6\% of the subjects with insufficient sleep (not insomnia) by overwork in Study 2 slept 6 hours or more on weekdays. In addition, it was revealed in Study 3 that some subjects who had objective symptoms of insufficient sleep were not aware of sleep loss. From our results, we concluded that short sleep 
duration, insomnia and insufficient sleep are different, although they can overlap, and must be analyzed separately. As shown in Fig. (3), insufficient sleep is a result of poor quantity of sleep caused by sleep deprivation or sleep restriction, whereas insomnia, that is poor quality of sleep, results from disturbance or disruption of sleep. Sleep debt might be a good indicator of insufficient sleep especially caused by overwork. Furthermore, assessing sleep health, including sleep debt, may be very important for promoting mental health. The mean sleep debt of individuals with sleep loss whether from insufficient sleep and/or insomnia was 86.6 minutes and that with insufficient sleep from overwork was 109.6 minutes, which may be a point of reference for risk of poor mental health. Future studies are needed to establish integrated guidelines for assessing sleep debt. In any case, we insist that sleep debts must be paid off.

\section{CONFLICT OF INTEREST}

There is no financial or other relationship that might lead to a conflict of interest.

\section{ACKNOWLEDGEMENTS}

We are grateful for the support of all of the staff at the Osaka Prefectural Mental Health Center, especially Toshiko Satoh, Satoko Ito, Momoko Nishitani, and Yukari Abe.

\section{REFERENCES}

[1] Liu X, Uchiyama M, Kim K, et al. Sleep loss and daytime sleepiness in the general adult population of Japan. Psychiatr Res 2000; 93: 1-11.

[2] Kaneita Y, Ohida T, Uchiyama M, et al. Excessive daytime sleepiness among the Japanese general population. J Epidemiol 2005; 15: $1-8$.

[3] Shimomitsu T, Levi L. Recent changes of working life in Japan. Eur J Public Health 1992; 2: 76-86.

[4] Amagasa T, Nakayama T, Takahashi Y. Karojisatsu in Japan: characteristics of 22 cases of work-related suicide. J Occup Health 2005; 47: 157-64.

[5] Iwasaki K, Takahashi M, Nakatani A. Health problems due to long working hours in Japan: Working hours, workers' compensation (Karoshi), and preventive measures. Industrial Health 2006; 44: 537-40.

[6] The Editorial Department of Occupational Health Journal. The enforcement of revised industrial safety and health law. Occup Health J 2006; 29: 4-11 (in Japanese).

[7] Hale L. Who has time to sleep? J Public Health 2005; 27: 205-11.

[8] Kageyama T, Nishikido N, Kobayashi T, Kawagoe H. Estimated sleep debt and work stress in Japanese white-collar workers. Psychiatry Clin Neurosci 2001; 55: 217-9.
[9] Léger D, Roscoat E, Bayon V, Guignard R, Pâquereau J, Beck F. Short sleep in young adults: insomnia or sleep debt? Prevalence and clinical description of short sleep in a representative sample of 1004 young adults from France. Sleep Med 2011; 12: 454-62.

[10] Buysse DJ, Reynolds CF 3rd, Monk TH, Berman SR, Kupfer DJ. The Pittsburgh Sleep Quality Index: a new instrument for psychiatric practice and research. Psychiatr Res 1989; 28: 193-213.

[11] Doi Y, Minowa M, Uchiyama M, et al. Psychometric assessment of subjective sleep using Japanese version of the Pittsburgh Sleep Quality Index (PSQI-J) in psychiatric disordered and control subjects. Psychiatr Res 2000; 97: 16572.

[12] Johns MW. A new method for measuring daytime sleepiness: the Epworth Sleepiness Scale. Sleep 1991; 14: 540-5.

[13] Goldberg, D. The Detection of Psychiatric Illness by Questionnaire. London: Oxford University Press 1972.

[14] Tanaka H, Shirakawa S. Sleep health, lifestyle and mental health in the Japanese elderly: ensuring sleep to promote a healthy brain and mind. J Psychosom Res 2004; 56: 465-77.

[15] Suzuki H, Kaneita Y, Osaki Y, et al. Clarification of the factor structure of the 12-item General Health Questionnaire among Japanese adolescents and associated sleep status. Psychiatr Res 2011; 188: $138-46$.

[16] Chen I, Vorona R, Chiu R, Ware JC. A survey of subjective sleepiness and consequences in attending physicians. Behav Sleep Med 2008; 6: 1-15.

[17] Regestein Q, Natarajan V, Pavlova M, Kawasaki S, Gleason R, Koff E. Sleep debt and depression in female college students. Psychiatr Res 2010; 176: 34-9.

[18] Prather AA, Marsland AL, Hall M, Neumann SA, Muldoon MF, Manuck SB. Normative variation in self-reported sleep quality and sleep debt is associated with stimulated pro-inflammatory cytokine production. Biol Psychol 2009; 82: 12-7.

[19] JapanVisitor.com. Suicide prevention campaign. May 21, 2010. http://japanvisitor.blogspot.jp/2010/05/suicidepreventioncampaign. html

[20] Kohyama J. More sleep will bring more serotonin and less suicide in Japan. Med Hypotheses 2010; 75: 340.

[21] Haavisto ML, Porkka-Heiskanen T, Hublin C, et al. Sleep restriction for the duration of a work week impairs multitasking performance. J Sleep Res 2010; 19: 444-54.

[22] Magee L, Hale L. Longitudinal associations between sleep duration and subsequent weight gain: A systematic review. Sleep Med Rev 2012; 16: 231-41.

[23] Knutson KL, Ryden AM, Mander BA, Van Cauter E. Role of sleep duration and quality in the risk and severity of type 2 diabetes mellitus. Arch Intern Med 2006; 166: 1768-74.

[24] Mezick EJ, Hall M, Matthews KA. Sleep duration and ambulatory blood pressure in black and white adolescents. Hypertension 2012; 59: 747-52.

[25] van Leeuwen WM, Lehto M, Karisola P, et al. Sleep restriction increases the risk of developing cardiovascular diseases by augmenting proinflammatory responses through IL-17 and CRP. PLoS One 2009; 4: e4589.

[26] Sabanayagam C, Shankar A. Sleep duration and cardiovascular disease: results from the National Health Interview Survey. Sleep 2010; 33: 1037-42.

[27] Grandner MA, Hale L, Moore M, Patel NP. Mortality associated with short sleep duration: The evidence, the possible mechanisms, and the future. Sleep Med Rev 2010; 14: 191-203. 